\title{
Expérience et simulation d'une décharge capillaire ablative pour la production de rayonnement UVX cohérent et incohérent
}

\author{
R. Dussart, S. Götze, D. Hong, J. Pons, C. Cachoncinlle, C. Fleurier, \\ J.-M. Pouvesle et M. Pöckl
}

GREMI, UMR 6606 du CNRS, Université d'Orléans, BP. 6744, 45067 Orléans cedex 2, France

\begin{abstract}
Résumé : Le code de calcul CADILAC a été utilisé pour simuler la dynamique de peuplement des états atomiques d'un plasma de carbone fortement ionisé. Le plasma est obtenu expérimentalement à partir d'une décharge capillaire de forte densité de puissance $\left(70 \mathrm{GW} \cdot \mathrm{cm}^{-3}\right)$. Le modèle permet de déterminer les domaines de paramètres potentiellement intéressants pour obtenir une amplification d'émission spontanée. Il confirme que des inversions de population sur plusieurs raies de Balmer du CVl peuvent se produire comme cela a été mis en évidence expérimentalement.
\end{abstract}

\section{INTRODUCTION}

Les décharges capillaires sont étudiées pour la création de sources UVX intenses incohérentes et cohérentes (laser X). En ce qui concerne les sources cohérentes, un dispositif expérimental a été installé au GREMI pour créer un plasma de carbone complètement ionisé, qui, en se recombinant, peut mener à une inversion de population entre deux niveaux du carbone hydrogénoïde CVI. Pour favoriser le processus de recombinaison à trois corps, le plasma doit subir une baisse brutale de la température électronique juste après sa complète ionisation. Si, en plus, la densité électronique est suffisamment élevée $\left(10^{19} \mathrm{~cm}^{-3}\right)$, les conditions requises sont alors remplies pour permettre le peuplement du niveau supérieur de la transition laser avec efficacité. La dynamique de peuplement des niveaux atomiques des ions CVI obtenus dans des décharges capillaires ablatives a été modélisée en utilisant le code de calcul CADILAC [1]. C'est un modèle collisionnel radiatif zéro dimension dépendant du temps. Il a été élaboré par M. Pöckl et utilisé pour simuler les plasmas obtenus expérimentalement. Après un rappel du dispositif expérimental, nous expliquons le fonctionnement du code de calcul CADILAC et nous présentons les résultats de la simulation.

\section{DISPOSITIF EXPERIMENTAL}

Un schéma du dispositif expérimental est présenté en référence [2]. L'énergie est stockée dans 50 capacités boutons de $2 \mathrm{nF}$ chacune portées à une tension pouvant atteindre $40 \mathrm{kV}$. On utilise un capillaire en polyéthylène $\left(\mathrm{CH}_{2}\right)_{\mathrm{n}}$ ayant une longueur de $1,6 \mathrm{~cm}$ et un diamètre de $1 \mathrm{~mm}$. Un courant impulsionnel de $50 \mathrm{kA}$ avec un temps de montée de $50 \mathrm{~ns}$ est injecté dans le capillaire. Il permet l'ablation des parois et l'ionisation des atomes du matériau ablaté pour former le plasma de carbone fortement ionisé. Pour étudier le rayonnement XUV, un spectromètre champ plan Jobin-Yvon PGMPGS500 a été mis en place. Il est équipé d'un réseau 800 traits $\mathrm{mm}^{-1}$. Le spectre est détecté par un intensificateur MCP obturable (ouverture $\sim 10 \mathrm{~ns}$ ) dont l'image en sortie est reprise par une caméra CCD intensifiée.

\section{PRESENTATION SUCCINCTE DU CODE DE CALCUL CADILAC}

Une présentation détaillée du code est donnée en référence [1]. La dynamique du plasma de décharge est modélisée par un ensemble d'équations différentielles non linéaires du premier ordre décrivant les échanges d'énergie entre le circuit électrique et la colonne de plasma. Trois groupes d'équations peuvent être distingués mettant en jeu trois types de variables : les variables de la décharge électrique, les variables $\mathrm{T}_{\mathrm{e}}$ et $\mathrm{N}_{\mathrm{e}}$ du plasma et les populations des différents niveaux atomiques pris en compte. Le circuit

Adresse actuelle : SIEMENS PSE, Gudrunstrasse 11, 1100 Wien, Autriche. 
électrique est modélisé par un circuit RLC simple série. Pour calculer la température électronique du plasma, un équilibre en énergie entre le chauffage ohmique et le refroidissement du plasma par rayonnement et par diffusion thermique est utilisé. La perte par rayonnement regroupe l'émission spontanée, la recombinaison radiative, et le rayonnement de freinage. Enfin, les densités de population relative sont calculées prenant en compte 6 niveaux héliumö̈des et 15 niveaux hydrogénö̈des. Les paramètres libres tels que le facteur d'ablation sont adaptés pour obtenir des valeurs de $T_{e}$ et $N_{e}$ compatibles avec celles obtenues expérimentalement après le maximum du courant.

\section{RESULTATS EXPERIMENTAUX ET COMPARAISON AUX RESULTATS DU CODE}

Des mesures de densité $\left(\mathrm{N}_{\mathrm{e}}\right)$ et de température électroniques $\left(\mathrm{T}_{\mathrm{c}}\right)$ ont été effectuées et publiées [3]. La densité électronique mesurée est de l'ordre de $10^{19} \mathrm{~cm}^{-3}$; la température électronique obtenue expérimentalement atteint $80 \mathrm{eV}$ juste après le maximum de courant. En figure 1, nous présentons l'évolution temporelle de $N_{e}$ et $T_{e}$ calculée par le code, ainsi que le courant expérimental. La température électronique calculée atteint une valeur maximale de $135 \mathrm{eV}$ au début de la décharge. Ceci est dû à la faible quantité de matière ablatée à cet instant. Ensuite, elle diminue jusqu'à atteindre environ $20 \mathrm{eV}$ à la fin de la première demi période du courant. Pour les mêmes conditions de décharge, nous présentons en figure 2 l'évolution des niveaux hydrogénoïdes de $n=2$ à $n=5$ obtenue avec le code CADILAC. Des inversions de population sur plusieurs raies de Balmer apparaissent. Une amplification avait déjà été observée expérimentalement sur les raies $\mathrm{Ha}$ et $\mathrm{H} \beta$ simultanément [2].

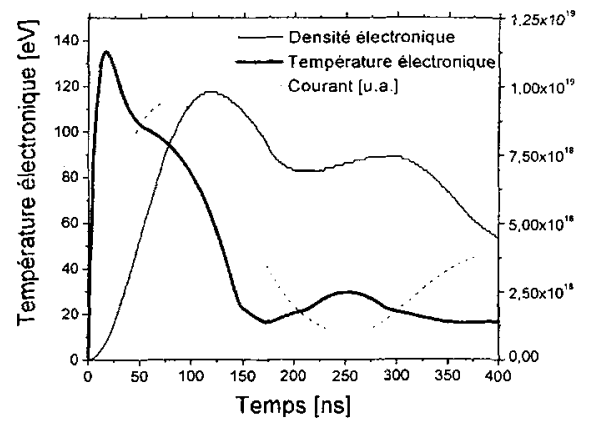

Fig1: Température et densité électroniques calculées par le code

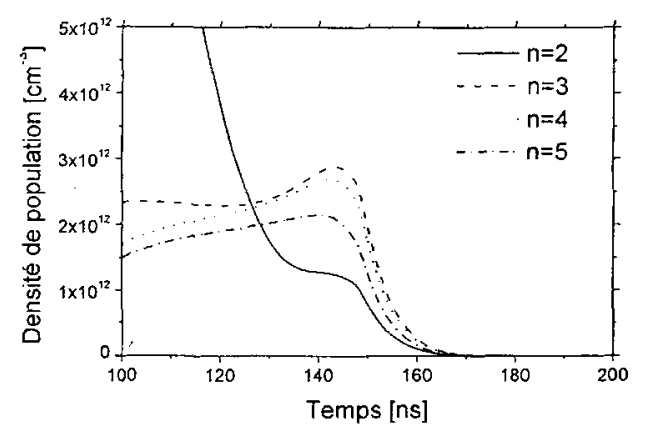

Fig2: Evolution des densités de population des niveaux du $\mathrm{C}$,

\section{CONCLUSION}

Le code CADILAC a été utilisé pour simuler la dynamique du plasma obtenu par décharge capillaire. Il montre que des inversions de population sur plusieurs raies de la série de Balmer peuvent être obtenues pendant le refroidissement brutal du plasma entraînant, de ce fait, une amplification d'émission spontanée possible sur plusieurs transitions comme nous l'avons observé expérimentalement.

Ce travail a reçu le soutien financier du réseau TMR FACADIX (contrat n ERBFMRXCT 980186)

\section{Références}

[1] M. Pöckl, M. Hebenstreit, T. Neger, F. Aumayr, J. Appl. Phys, 76 (2), 733, 1994.

[2] R. Dussart, W. Rosenfeld, N. Richard, D. Hong, C. Cachoncinlle, C. Fleurier, J.-M. Pouvesle, in X-Ray Lasers 1998, Proceedings of the $6^{\text {th }}$ International Conference on X-Ray Lasers. 171, 1999.

[3] D. Hong, R. Dussart, C. Cachoncinlle, W. Rosenfeld, S. Götze, J. Pons, R. Viladrosa, C. Fleurier, J.-M. Pouvesle, Rev. Sci. Ins., 71 (1), 15, 2000. 